\title{
Hypothalamo-pituitary-adrenal axis dysregulation in patients with rheumatoid arthritis after the dexamethasone/corticotrophin releasing factor test
}

\author{
MS Harbuz, E Korendowych ${ }^{1}$, DS Jessop, AL Crown, SL Lightman \\ and JR Kirwan ${ }^{1}$ \\ University Research Centre for Neuroendocrinology and 'Department of Rheumatalogy, University of Bristol, Bristol Royal Infirmary, Marlborough Street, \\ Bristol BS2 8HW, UK \\ (Requests for offprints should be addressed to M Harbuz; Email: m.s.harbuz@bristol.ac.uk)
}

\begin{abstract}
A defective hypothalamo-pituitary-adrenal axis response to inflammatory cytokines may contribute to the pathophysiology of rheumatoid arthritis (RA). The purpose of this study was to define further the mechanisms responsible for this dysregulation. Six normal individuals and seven patients with active RA were recruited and given an oral dose of dexamethasone at $2300 \mathrm{~h}$ the evening before the study. The next day, an i.v. catheter was fitted at $1300 \mathrm{~h}$. Blood samples were collected between $1400 \mathrm{~h}$ and $1700 \mathrm{~h}$ before and after infusion (at $1500 \mathrm{~h}$ ) of corticotrophin releasing factor (CRF). Plasma was separated and stored at $-20{ }^{\circ} \mathrm{C}$ before radioimmunoassay for ACTH, cortisol and dihydroepiandrosterone (DHEA). Before the CRF challenge, ACTH and cortisol were significantly increased and DHEA significantly decreased in the
\end{abstract}

patients with RA compared with the controls. Neither ACTH nor DHEA was significantly altered after CRF infusion. Control individuals did not mount a cortisol response to infusion of CRF. Similarly, four of the patients with RA did not respond to CRF. However, in contrast to the controls, three of the patients mounted an immediate and sustained cortisol response after receiving CRF. These data reveal that three of the seven patients with RA were able to escape from dexamethasone suppression and mount a cortisol response to CRF challenge. This suggests that there may be a subpopulation of patients with RA who have impaired glucocorticoid feedback. The implications of this alteration for disease progression remain to be determined.

Journal of Endocrinology (2003) 178, 55-60

\section{Introduction}

Rheumatoid arthritis (RA) is an immune-mediated inflammatory condition. It is characterised by joint pain, swelling and stiffness through inflammation in the synovial joint lining, with increasing joint impairment and disability as a result of gradual erosion of the bone and cartilage of the joints. The pathogenesis or progression of the disease is influenced by a combination of genetic and environmental factors, although the precise mechanisms remain obscure. In recent years, attention has focused on the interactions of neuroendocrine and immune components of the inflammatory response in regulating the onset and severity of RA. Both basic and clinical research have identified the hypothalamo-pituitary-adrenal (HPA) axis as being of potential importance in regulating disease activity.

At the hypothalamic level, activity of the HPA axis is co-ordinated by corticotrophin releasing factor (CRF) neurones located in the parvocellular cells of the paraventricular nucleus (PVN). Under normal conditions in man and in rodents, approximately 50\% of these CRF neurones also contain arginine vasopressin (AVP) (Erkut et al. 1995, Harbuz \& Lightman 1997). After a variety of challenges there is an increase in the number of CRF cells co-expressing AVP (Erkut et al. 1995, Harbuz \& Lightman 1997). Axons from these parvocellular neurones terminate in the external zone of the median eminence, where CRF and AVP are released into the hypophysial portal blood and carried to the corticotrophs of the anterior pituitary. CRF is currently the only factor shown to increase the levels of mRNA for pro-opiomelanocortin (POMC), the adrenocorticotrophic hormone (ACTH) precursor. CRF and AVP exert a synergistic action on the release of ACTH into the general circulation. ACTH causes the synthesis and release of cortisol from the adrenal cortex, and cortisol exerts a negative feedback action at the pituitary, hypothalamus and other central areas, to maintain homeostasis (Harbuz \& Lightman 1997).

The HPA axis is activated in response to stress and also in response to acute immune challenge - for example, from lipopolysaccharide (LPS) or cytokines such as tumour 
necrosis factor- $\alpha$ (TNF $\alpha$ ), interleukin (IL)-1 and IL-6 (Harbuz \& Lightman 1997). After such acute challenges, there is an activation at all levels of the axis, resulting in the release of glucocorticoids from the adrenal cortex; in man it is cortisol that is released. These glucocorticoids exert a negative feedback action at the level of the pituitary, hypothalamus and other higher brain centres to regulate further activation. In addition, glucocorticoids have a potent immunosuppressive effect, preventing further immune stimulation of the HPA axis and overactivity of the immune system. This anti-inflammatory action is crucial in chronic inflammatory disease conditions. Blocking glucocorticoid synthesis with metyrapone results in a sudden flare in disease activity in patients with RA (Saldanha et al. 1986).

The integrity of the HPA axis in humans with RA is less clear, with some workers reporting major alterations whereas others believe that dysfunction may be more subtle (Harbuz \& Jessop 1999). However, AVP is believed to have an important role in glucocorticoid regulation in RA (Chikanza et al. 2000). Other hormones are also abnormally regulated in RA. In particular, dehydroepiandrosterone (DHEA) and the sulphated form, DHEA-S, have been reported to be decreased in both RA and systemic lupus erythematosus (Hedman et al. 1989, Masi et al. 1999, 2000, Dessein et al. 2001). These adrenal steroids are the most abundant among those found in the human circulation. DHEA has been reported to inhibit secretion of proinflammatory cytokines such as IL-6 and TNFa in vitro (Rook et al. 1994, Di Santo et al. 1996, Straub et al. 1998). The naturally occurring decrease in androgen secretion associated with ageing and the increased incidence of autoimmune disease in aged individuals make DHEA a suitable target for investigation.

The dexamethasone/CRF test has been used extensively to assess HPA axis dysfunction in a variety of psychiatric disorders (Von Bardeleben \& Holsboer 1991, Steckler et al. 1999). Recently this test, which eliminates cortisol from the feedback control loop, has been extended to patients with multiple sclerosis (Kumpfel et al. 1999). In the present study we have determined concentrations of DHEA, cortisol and ACTH after the dexamethasone/ CRF test in control individuals and in patients with active RA.

\section{Participants and Methods}

\section{Participants}

Seven patients (six women, one man: mean age $51 \pm$ (S.E.M.) 3.5 years; mean disease duration $16 \pm 4$ years), with seropositive RA who were being admitted to hospital because of a flare of their disease, and six controls (five women, one man: mean age $52 \pm 2$ years), were recruited to the study. None of the participants had received corticosteroids, either orally or by injection, for at least 3 months before the study. All the patients were taking non-steroidal anti-inflammatory drugs.

\section{Procedure}

Ethics approval for this study was obtained from the Research Ethics Committee of the United Bristol Healthcare NHS Trust. Written informed consent was obtained from all participants in the study. Patients with active, widespread joint inflammation were admitted to the Rheumatology Unit for bed rest. On the day of admission they were asked to complete a Health Assessment Questionnaire (HAQ) and a formal clinical assessment was made of the state of their arthritis (number of tender and swollen joints). In addition to their usual medications, they were given $1.5 \mathrm{mg}$ dexamethasone orally at $2300 \mathrm{~h}$. The next day they remained ambulant and were given breakfast and lunch. At $1300 \mathrm{~h}$ they commenced bed rest and an i.v. catheter was fitted. Baseline blood samples $(5 \mathrm{ml})$ were taken at $1400 \mathrm{~h}$ and $1430 \mathrm{~h}$. At $1500 \mathrm{~h}, 100 \mu \mathrm{g}$ human CRF was infused through the catheter over a period of $30 \mathrm{~s}$. Further blood samples were withdrawn at 15-min intervals over the next $2 \mathrm{~h}$. Control individuals were given $1.5 \mathrm{mg}$ dexamethasone to be taken at $2300 \mathrm{~h}$ and were asked to report for CRF and blood sampling at $1300 \mathrm{~h}$ the next day.

\section{Blood samples and radioimmunoassays}

Blood samples $(5 \mathrm{ml})$ were placed on ice in EDTA tubes immediately after collection. They were then centrifuged and the plasma separated, aliquoted and frozen at $-20{ }^{\circ} \mathrm{C}$ before measurement of $\mathrm{ACTH}$ and cortisol using in-house radioimmunoassays, and DHEA using a radioimmunoassay kit (ICN, Boldon, Tyne and Wear, UK).

\section{Data analysis}

Means and 95\% confidence intervals (CI) or S.E.M. were calculated for the clinical descriptions and for plasma cortisol, ACTH, DHEA and the cortisol:DHEA ratio. The cortisol, ACTH and DHEA responses to i.v. CRF were inspected for each participant and each was designated a 'responder' or 'non-responder' according to the pattern observed. Statistical analysis was made using the unpaired Student's $t$-test.

\section{Results}

Baseline plasma concentrations of ACTH, cortisol and DHEA after oral dexamethasone are shown in Table 1. According to the standard criteria, in the dexamethasone suppression test plasma cortisol concentrations were less than $40 \mathrm{ng} / \mathrm{ml}$ in all patients and controls (except one who was excluded as having extremely high cortisol and 
Table 1 Plasma cortisol, ACTH and DHEA concentrations in control subjects and patients with RA after dexamethasone but before the CRF test. Values are presented as the mean with 95\% Cl.

\begin{tabular}{|c|c|c|c|}
\hline & Mean & $95 \% \mathrm{Cl}$ & $P$ \\
\hline \multicolumn{4}{|c|}{ Cortisol (ng/ml) } \\
\hline Controls & 12 & 7 to 9 & \\
\hline Patients & 22 & 15 to 29 & 0.037 \\
\hline \multicolumn{4}{|c|}{ АCTH (pg/ml) } \\
\hline Controls & 10 & 9 to 11 & \\
\hline Patients & 56 & 50 to 62 & $<0.0001$ \\
\hline \multicolumn{4}{|c|}{ DHEA (ng/ml) } \\
\hline Controls & 625 & 359 to 891 & \\
\hline Patients & 228 & 122 to 334 & $0 \cdot 044$ \\
\hline
\end{tabular}

DHEA concentrations: cortisol $143 \mathrm{ng} / \mathrm{ml}$, DHEA $1706 \mathrm{ng} / \mathrm{ml})$. There was a significant increase in plasma concentrations of ACTH $(P<0 \cdot 0001)$ and cortisol $(P=$ $0.037)$ in patients with RA compared with controls. In contrast, DHEA concentrations were significantly $(P=$ 0.044) reduced in the patients with RA.

The cortisol responses to i.v. CRF in the controls and in individual patients with RA are shown in Fig. 1. Control participants did not respond to the CRF challenge, although one did show a short-lived increase in plasma cortisol at 60 and $75 \mathrm{~min}$ after receiving CRF. At this late time after CRF infusion, this increase was not considered to be a response to CRF. The data from this patient are included in the mean values presented in Fig. 1. Four of the patients with RA did not respond to CRF and, in contrast to the controls, three of the patients showed an immediate and sustained increase in plasma cortisol.

Individual scores for all the patients for the HAQ, numbers of tender and swollen joints and serum C-reactive protein concentrations are shown in Table 2. They show patients with moderately severe disease and currently active joint inflammation. There was no significant difference between responders and non-responders for any of these parameters measured.

Neither ACTH nor DHEA was significantly altered in response to CRF challenge after dexamethasone pretreatment. Similarly, none of the patients with RA showed any alteration in plasma ACTH or DHEA in response to CRF.

Comparing the groups for baseline cortisol:DHEA ratios before CRF infusion revealed a significant $(P<0 \cdot 01)$ difference between the controls (mean 24.1; 95\% CI $15 \cdot 2$ to $33 ; n=5$ ) and patients with RA (mean $8 \cdot 6 ; 95 \%$ CI $3 \cdot 2$ to $14 ; n=7)$. The ratio was further reduced when the non-responders were removed from the analysis (mean $5 \cdot 9$; CI $2 \cdot 2$ to $9 \cdot 6 ; n=3$ ), although this was not significantly different from the group value for all the patients with RA who were tested.

\section{Discussion}

This is the first time that the dexamethasone/CRF test has been used in patients with RA. A striking feature of these data is the finding that three of the seven patients with RA were able to escape from dexamethasone suppression and mount a cortisol response to the CRF challenge. In contrast, and as expected, none of the controls was able to generate such a response. In addition, plasma concentrations of cortisol and ACTH were significantly increased in the patients, and DHEA concentrations were significantly decreased. The baseline cortisol:DHEA ratio before the administration of CRF was also significantly decreased in the patients with RA, in agreement with previously published data (Straub et al. 2002). Together, these data suggest an abnormal regulation of the HPA axis in a subpopulation of patients with RA. The lack of any significant increase in ACTH in the patients who mounted a cortisol response may be attributable to a number of factors; possible candidates might be POMC fragments, an alteration in adrenal sensitivity, or an increased sympathetic nervous system activity at the adrenal medulla that modulates cortisol release. Before the administration of CRF, the ACTH:cortisol ratio was threefold greater in the patients compared with that in the controls ( 2.55 compared with 0.83 respectively), suggesting hyposensitivity to ACTH at the level of the adrenal cortex in patients with RA. Further studies are needed to address the responsiveness of the ACTH-independent systems to CRF.

Significantly increased baseline concentrations of ACTH and cortisol after dexamethasone and before the administration of CRF were evident in the patients with RA compared with the controls; we did not determine plasma concentrations of these hormones before the administration of dexamethasone. This increase in baseline concentration may suggest that dexamethasone was less effective at suppressing plasma cortisol in the patients with RA than in the controls. The role of the HPA axis in RA has recently been reviewed, leading to the conclusion that most studies to date have not revealed major differences in HPA axis activity in patients with RA compared with that in control individuals (Harbuz \& Jessop 1999). However, subtle differences may exist that influence the time course or severity of inflammation. Comparison of controls and patients with RA after dexamethasone with and without CRF challenge may help elucidate these differences.

The present data confirm the findings of previous studies in which normal individuals have consistently been shown to be unable to respond to CRF after dexamethasone pretreatment. In contrast, patients with depression are able to respond to CRF in this context, suggestive of abnormal regulation of the HPA axis in these individuals (Von Bardeleben \& Holsboer 1991, Steckler et al. 1999). The mechanisms underlying this escape in patients with depression are unknown, but it has been suggested 


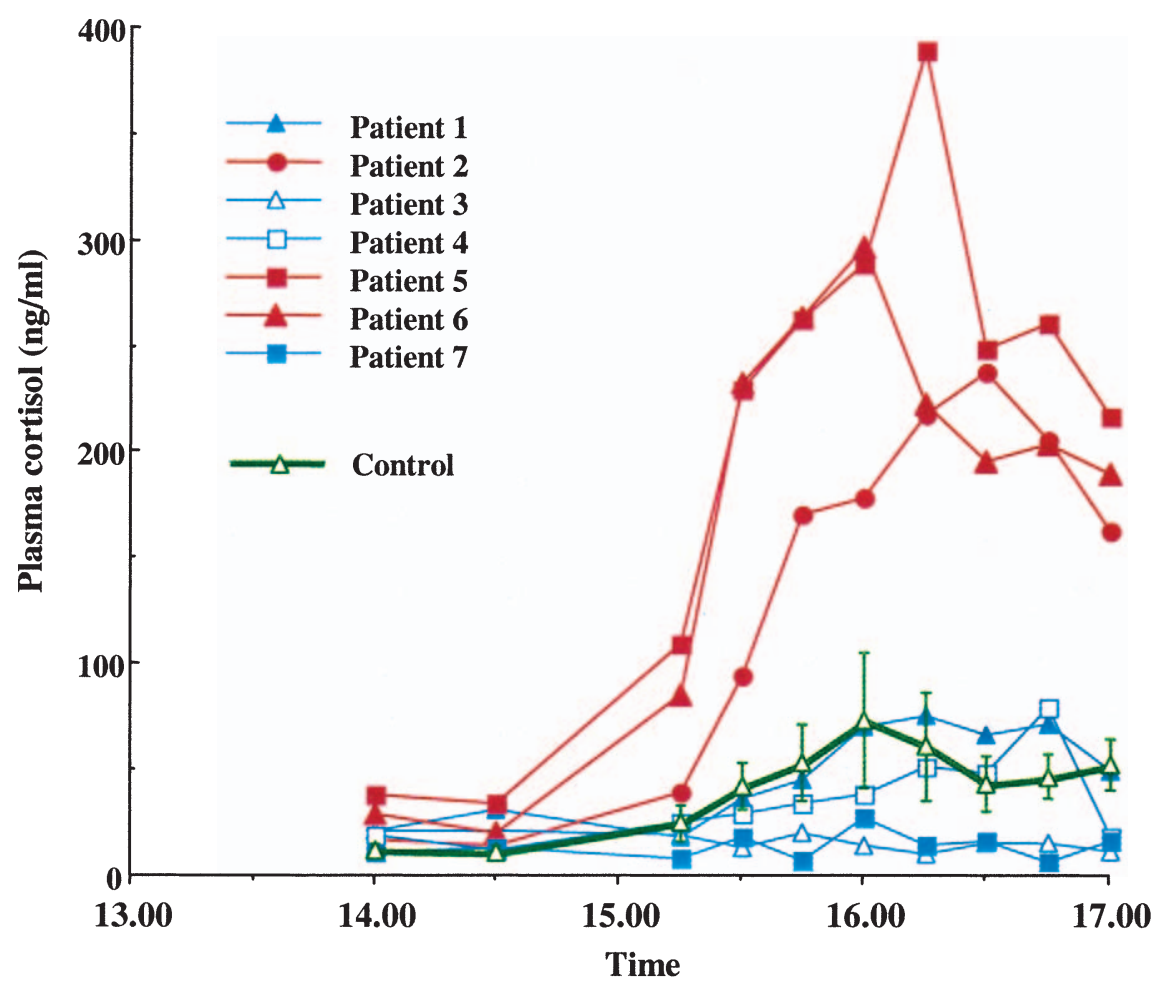

Figure 1 Plasma cortisol $(\mathrm{ng} / \mathrm{ml})$ in control individuals (thick line; mean \pm S.E.M. for $n=6$ individuals) and individual patients with RA $(n=7)$ in the dexamethasone/CRF test. Time represents clock time, beginning at $1300 \mathrm{~h}$. CRF was infused at $1500 \mathrm{~h}$.

that there is a shift in the dose-response curve towards decreased glucocorticoid receptor sensitivity in depressed patients, reflecting feedback resistance at the level of the PVN and pituitary (Modell et al. 1997). The involvement of corticosteroid receptors is supported by the evidence of decreased hippocampal mineralocorticoid and glucocorticoid receptor mRNA levels in suicide victims with a

Table 2 Health assessment questionnaire (HAQ) score, number of tender and swollen joints and C-reactive protein concentrations (CRP) in the patients with RA. The patients are grouped according to whether they were able to mount a cortisol response to CRF challenge (Responders) or not (Non-responders).

\begin{tabular}{|c|c|c|c|}
\hline HAQ score & $\begin{array}{l}\text { Tender } \\
\text { (No.) }\end{array}$ & $\begin{array}{l}\text { Swollen } \\
\text { (No.) }\end{array}$ & $\begin{array}{l}\text { CRP } \\
\text { (mg/L units) }\end{array}$ \\
\hline
\end{tabular}

Patient No.

Non-responders

\begin{tabular}{llrrr}
1 & $2 \cdot 25$ & 17 & 17 & 32 \\
3 & $2 \cdot 125$ & 16 & 11 & 60 \\
4 & $2 \cdot 25$ & 8 & 6 & 17 \\
7 & $1 \cdot 75$ & 26 & 15 & 20 \\
Responders & & & & \\
2 & $1 \cdot 75$ & 12 & 6 & 10 \\
5 & $2 \cdot 25$ & 8 & 4 & 32 \\
6 & $1 \cdot 75$ & 10 & 5 & 22 \\
\hline
\end{tabular}

history of depression (Lopez et al. 1998). Alcohol abusers who are undergoing withdrawal also exhibit increased cortisol concentrations in response to the dexamethasone/ CRF test. After successful withdrawal of alcohol, the cortisol response to CRF was normal (Hundt et al. 2001). At the level of the PVN, the activity of the HPA axis may be mediated by AVP that is synthesised in a subpopulation of CRF cells within the PVN. An increase in the number of CRF cells co-localising AVP has been reported in postmortem studies of patients with depression (Purba et al. 1996). An increase in AVP activity has also been reported in rats with adjuvant-induced arthritis (Harbuz et al. 1992, Chowdrey et al. 1995). These data suggest that abnormal HPA axis regulation may be a feature of other chronic illnesses besides psychiatric disease, but that this may not reflect an inherent defect in HPA axis regulation, as HPA axis activity appears to be normal in patients who recover from illness.

Recently, the dexamethasone/CRF test has been used to investigate abnormal HPA axis regulation in patients with the autoimmune disease, multiple sclerosis (MS). These patients exhibited an exaggerated increase in plasma cortisol on challenge with CRF that was dependent on the clinical course of the MS. Those with relapsing-remitting disease had a modest increase in cortisol, those with secondary progressive MS were intermediate, and the 
greatest response was seen in patients with chronic primary progressive MS (Kumpfel et al. 1999, Then Bergh et al. 1999). In the present study, all the RA patients were admitted to hospital as a consequence of a flare of their disease. We were unable to determine any differences in terms of age, HAQ scores or measures of severity of inflammation (tender or swollen joint counts or C-reactive protein concentrations) between the responders and nonresponders. Although we did not rate the degree of depressed mood, none of our patients was receiving treatment for depression. No correlation with depressed mood was found in the MS study (Then Bergh et al. 1999), suggesting that depression is unlikely to be a factor explaining the observations in the responsive group of patients with RA.

In a study of patients with remission from depression, an increased cortisol response was associated with a greater risk of relapse (Zobel et al. 2001). In the present investigation, we were unable to study our patients over a long follow-up. It would be intriguing in future studies to determine if there were any long-term alterations in disease activity or severity, comparing the responders and the non-responders to the dexamethasone/CRF test.

This paper is the first to report that a subpopulation of patients with RA are able to escape the effects of dexamethasone suppression and mount a cortisol response to CRF challenge. We were unable to determine any immediate differences in disease severity between the responders and the non-responders, but these differences may emerge when larger numbers are identified and the course of their disease is studied in more detail. Further investigation will be required to assess the underlying mechanisms and the implications of these differences.

\section{Acknowledgement}

We would like to thank Dr M Sharif for measurement of C-reactive protein concentrations.

\section{References}

Chikanza IC, Petrou P \& Chrousos G 2000 Perturbations of arginine vasopressin secretion during inflammatory stress. Pathophysiologic implications. Annals of the New York Academy of Sciences 917 825-834.

Chowdrey HS, Larsen PJ, Harbuz MS, Jessop DS, Aguilera G, Eckland DJ \& Lightman SL 1995 Evidence for arginine vasopressin as the primary activator of the HPA axis during adjuvant-induced arthritis. British Journal of Pharmacology 116 2417-2424.

Dessein PH, Joffe BI, Stanwix AE \& Moomal Z 2001 Hyposecretion of the adrenal androgen dehydroepiandrosterone sulfate and its relation to clinical variables in inflammatory arthritis. Arthritis Research 3 183-188.

Di Santo E, Foddi MC, Ricciardi-Castagnoli P, Mennini T \& Ghezzi P 1996 DHEAS inhibits TNF production in monocytes, astrocytes and microglial cells. Neuroimmunomodulation 3 285-288.
Erkut ZA, Hofman MA, Ravid R \& Swaab DF 1995 Increased activity of hypothalamic corticotropin-releasing hormone neurons in multiple sclerosis. Journal of Neuroimmunology 62 27-33.

Harbuz MS \& Jessop DS 1999 Is there a defect in cortisol production in rheumatoid arthritis? Rheumatology 38 298-302.

Harbuz MS \& Lightman SL 1997 Signals from the hypothalamus to the pituitary during chronic immune responses. In: Steroid Hormones and the T-Cell Cytokine Profile, pp 15-52. Eds GAW Rook \& SL Lightman. London: Springer-Verlag.

Harbuz MS, Rees RG, Eckland D, Jessop DS, Brewerton D \& Lightman SL 1992 Paradoxical responses of hypothalamic corticotropin-releasing factor (CRF) messenger ribonucleic acid (mRNA) and CRF-41 peptide and adenohypophysial proopiomelanocortin $\mathrm{mRNA}$ during chronic inflammatory stress. Endocrinology 130 1394-1400.

Hedman M, Nilsson E \& de la Torre B 1989 Low sulpho-conjugated steroid hormone levels in systemic lupus erythematosus (SLE). Clinical and Experimental Rheumatology 7 583-588.

Hundt W, Zimmermann U, Pottig M, Spring K \& Holsboer F 2001 The combined dexamethasone-suppression/CRH-stimulation test in alcoholics during and after acute withdrawal. Alcoholism, Clinical and Experimental Research 25 687-691.

Kumpfel T, Then Bergh F, Friess E, Uhr M, Yassouridis A, Trenkwalder C \& Holsboer F 1999 Dehydroepiandrosterone response to the adrenocorticotropin test and the combined dexamethasone and corticotropin-releasing hormone test in patients with multiple sclerosis. Neuroendocrinology 70 431-438.

Lopez JF, Chalmers DT, Little KY \& Watson SJ 1998 Regulation of serotonin1A, glucocorticoid, and mineralocorticoid receptor in rat and human hippocampus: implications for the neurobiology of depression. Biological Psychiatry 43 547-573.

Masi AT, Chatterton RT \& Aldag JC 1999 Perturbations of hypothalamic-pituitary-gonadal axis and adrenal androgen functions in rheumatoid arthritis: an odyssey of hormonal relationships to the disease. Annals of the New York Academy of Sciences 876 53-62.

Masi AT, Aldag JC, Chatterton RT, Adams RF \& Kitabchi AE 2000 Adrenal androgen and glucocorticoid dissociation in premenopausal rheumatoid arthritis: a significant correlate or precursor to onset? Zeitschrift für Rheumatologie 59 (Suppl 2) II/54-61.

Modell S, Yassouridis A, Huber J \& Holsboer F 1997 Corticosteroid receptor function is decreased in depressed patients. Neuroendocrinology 65 216-222.

Purba JS, Hoogendijk WJ, Hofman MA \& Swaab DF 1996 Increased number of vasopressin- and oxytocin-expressing neurons in the paraventricular nucleus of the hypothalamus in depression. Archives of General Psychiatry 53 137-143.

Rook GA, Hernandez-Pando R \& Lightman SL 1994 Hormones, peripherally activated prohormones and regulation of the Th1/Th2 balance. Immunology Today 15 301-303.

Saldanha C, Tougas G \& Grace E 1986 Evidence for anti-inflammatory effect of normal circulating plasma cortisol. Clinical and Experimental Rheumatology 4 365-366.

Steckler T, Holsboer F \& Reul JM 1999 Glucocorticoids and depression. Bailliere's Best Practice and Research Clinical Endocrinology and Metabolism 13 597-614.

Straub RH, Konecna L, Hrach S, Rothe G, Kreutz M, Scholmerich J, Falk W \& Lang B 1998 Serum dehydroepiandrosterone (DHEA) and DHEA sulfate are negatively correlated with serum interleukin-6 (IL-6), and DHEA inhibits IL-6 secretion from mononuclear cells in man in vitro: possible link between endocrinosenescence and immunosenescence. Journal of Clinical Endocrinology and Metabolism 83 2012-2017.

Straub RH, Paimela L, Peltomaa R, Scholmerich J \& Leirisalo-Repo M 2002 Inadequately low serum levels of steroid hormones in relation to interleukin-6 and tumor necrosis factor in untreated patients with early rheumatoid arthritis and reactive arthritis. Arthritis and Rheumatism 46 654-662. 
Then Bergh F, Kumpfel T, Trenkwalder C, Rupprecht R \& Holsboer F 1999 Dysregulation of the

hypothalamo-pituitary-adrenal axis is related to the clinical course of MS. Neurology 53 772-777.

Von Bardeleben U \& Holsboer F 1991 Effect of age on the cortisol response to human corticotropin-releasing hormone in depressed patients pretreated with dexamethasone. Biological Psychiatry 29 $1042-1050$.
Zobel AW, Nickel T, Sonntag A, Uhr M, Holsboer F \& Ising M 2001 Cortisol response in the combined dexamethasone/CRH test as predictor of relapse in patients with remitted depression. A prospective study. Journal of Psychiatric Research 35 83-94.

Received 24 December 2002

Accepted 27 March 2003 